\title{
Medical Problems Among Rohingya Refugees and Medications Prescribed During IMARET Clinic Visits in Kelantan
}

\author{
Tan Wen Rui ${ }^{1}$, Ahmad Rashidi Mohamed Tahir ${ }^{2,3}$, Nur Aizati Athirah Daud ${ }^{1}$ \\ ${ }^{1}$ School of Pharmaceutical Sciences, UniversitiSains Malaysia, Penang; ${ }^{2}$ Faculty of \\ Pharmacy, University of Cyberjaya, Selangor; ${ }^{3}$ IMAM Response \& Relief Team (IMARET), \\ Kuala Lumpur, Malaysia \\ DOI: http://dx.doi.org/10.31344/ijhhs.v5i0.317
}

Introduction: For years, thousands of the Rohingya have fled Myanmar to reside in different states in Malaysia. Without any legal status, they have limited access to government medical and healthcare facilities. This is because Malaysia is not a State party to the 1951 Refugee Convention and its 1967 Protocol. IMAM Response and Relief Team (IMARET), a medical NGO, has organized charity clinics for this minority group to provide basic medical aids.

Objectives: To identify common medical problems and medications prescribed during IMARET charity clinics in Kelantan.

Methods: This was a cross-sectional study among Rohingya refugees attending IMARET charity clinics in Kelantan from June 2017 until May 2018. Data was collected from a medical clerking sheet. Medical problems were classified using ICD-10 classification, while medications prescribed were classified according to the first level of ATC code. Data were analysed using SPSS Version 24.0.

Results: From 315 patients attended the clinic, most of the patients are of male gender $(n=232$, $73.1 \%$ ). Mean age of the patients was $21.9+13.6$ years (range: $1-75$ ). Patients attended either one of the clinic locations; Tanah Merah ( $\mathrm{n}=153,48.5 \%)$, PengkalanChepa $(\mathrm{n}=78,24.8 \%)$, and Kok Lanas $(\mathrm{n}=84,26.6 \%)$. Most common medical problems observed were related to musculoskeletal system $(\mathrm{n}=104,19.9 \%)$, mostly involving myalgia $(\mathrm{n}=96,30.5 \%)$. Other common problems involved respiratory system $(n=94,18.0 \%)$, digestive system $(n=68,13.0 \%)$, and skin problems $(\mathrm{n}=57,10.9 \%)$. Medications used for gastrointestinal disorders were mostly being prescribed in the clinics $(n=179,56.8 \%)$, followed by medications used for nervous system $(n=114,36.2 \%)$ and respiratory system $(n=97,30.8 \%)$. Most frequently prescribed medications include paracetamol, diclofenac, chlorpheniramine and multivitamins.

Conclusion: Common medical problems observed among Rohingya refugees include myalgia, respiratory diseases and gastrointestinal problems. Therefore, medications used to treat these problems were shown to be frequently prescribed during clinics.

Keywords: Rohingya refugee, IMARET, mobile clinic, medical problems, prescribed medication 